http://jmscr.igmpublication.org/home/ ISSN (e)-2347-176x ISSN (p) 2455-0450 crossref DOI: https://dx.doi.org/10.18535/jmscr/v8i3.20

\title{
Role of transvaginal gray scale and color doppler ultrasound imaging in ovarian lesions
}

Authors

\author{
Naresh Chandra Yadav ${ }^{1}$, Ashok Kumar Verma ${ }^{2 *}$, Rashmi $^{3}$, Rakesh Kumar Verma ${ }^{4}$ \\ ${ }^{1}$ Professor, ${ }^{2}$ Assistant Professor, ${ }^{3}$ Junior Resident, ${ }^{4}$ Junior Resident \\ Department of Radiodiagnosis GSVM medical college Kanpur U.P. India \\ *Corresponding Author \\ Dr Ashok Kumar Verma \\ Assistant Professor, Department of Radiodiagnosis, GSVM Medical College Kanpur U.P. India
}

\begin{abstract}
Introduction: The presence of an ovarian mass is one of the leading indications for gynaecological surgery. Ultrasonographyis considered the primary imaging modality for confirmation of the ovarian origin of the mass and characterization of the nature of the mass as benign or malignant. Transvaginal sonography unquestionably provides excellent depiction of the pelvic organs. It has been used as a routine technique as a part of the gynaecological examination. when morphologic features alone are applied to the prediction of ovarian malignancy, there is a tendency to over diagnose malignant tumors because of a substantial overlap between malignant and benign appearing masses. In addition, transvaginal color Doppler imaging with pulsed Doppler spectral analysis improves the characterization of ovarian masses by means of quantitative blood flow measurements obtained from tumor vessels.

Material \& Methods: This is a prospective study conducted in the Department of Radiodiagnosis, G.S.V.M. Medical College \& L.L.R Hospital, Kanpur, from December 2018 to November 2019. The study was conducted in collaboration with department of Obstetrics \& Gynecology. The study was conducted on 50 patients with 53 masses (three have bilateral masses). Patients were selected from the outpatient department and from admissions in ward. All adnexal masses were subjected to sono-morphological evaluation followed by blood flow analysis using color, spectral and power Doppler sonography. Morphologic indexing of the adnexal masses was done using the Sassone score based on the visualization of inner wall structure and wall thickness, septae, solid parts and echogenicity. On spectral Doppler, the lowest RI, PI and maximum PSV detected at any point in the mass was used for analysis. The Doppler findings were considered as being suggestive of malignancy when: RI $<0.6$, PI $<1$ and $P S V \max >15 \mathrm{~cm} / \mathrm{sec}$.

Results: 22 out of 53 masses were considered suspicious of malignancy (Sassone score $\geq 9$ ) of which 15 were malignant and 7 were benign in 31 mass lesions- 29 were benign and 2 were malignant. Malignant neoplasm offered lower resistance to blood flow due to presence of aberrant tumor vessels. In the present study $94.11 \%$ of malignant tumors showed RI $<0.6$ in contrast to only $11.11 \%$ of benign tumor. PSV was more than $15 \mathrm{~cm} / \mathrm{sec}$ in 15 (88.23\%) malignant tumors as compared to only $5.55 \%$ of benign tumors.

Conclusion: In conclusion to our study, there is considerable overlap in the morphologic patterns of various adnexal masses and hence a multi-parameter analysis incorporating morphologic scoring, vessel location, vessel arrangement and spectral waveform analysis is helpful in determining those patients in whom early intervention is necessary. Adding color Doppler to conventional sonography produced a specificity and positive predictive value higher than those of conventional sonography alone.

Keywords: Ultrasonograghy, Power Doppler sonography, Pulsality index, Resistance index.
\end{abstract}




\section{Introduction}

The presence of an ovarian mass is one of the leading indications for gynaecological surgery. Determination of a degree of suspicion for malignancy is critical and is based largely on imaging appearance. Ultrasonography is considered the primary imaging modality for confirmation of the ovarian origin of the mass and characterization of the nature of the mass as benign or malignant.

The introduction of high-frequency transvaginal ultrasonography in the mid-1980s, has allowed detailed morphologic evaluation of ovarian lesions. Transvaginalsonography unquestionably provides excellent depiction of the pelvic organs. It has been used as a routine technique as a part of the gynaecological examination.

However, when morphologic features alone are applied to the prediction of ovarian malignancey, there is a tendency to overdiagnose malignant tumors because of a substantial overlap between malignant and benigh-appearing masses. In addition, transvaginal color Doppler imaging with pulsed Doppler spectral analysis improves the characterization of ovarian masses by means of quantitative blood flow measurements obtained from tumor vessels. Color Doppler US helps identify solid, vascularized components in a mass. Spectral Doppler waveform characteristics correlate well with malignancy but generally add little information to morphologic considerations.

Adnexal masses are frequently found in both symptomatic and asymptomatic women. In premenopausal women, physiologic follicular cysts and corpus luteum cysts are the most common adnexal masses.

Other masses in this age group include endometrimas, polycystic overies, tubo-ovarian abscesses and benign neoplasms.

Malignant neoplasms are uncommon in younger women but become more frequent with increasing age. In postmenopausal women with adnexal masses, both primary and secondary neoplasms must be considered, along with leiomyomas, ovarian fibromas and other lesions such as diverticular abscesses.

Information from the history, physical examination, ultrasound evaluation and selected laboratory tests will enable the physician to find the most likely cause of an adnexal mass.

Computed tomography can help assess the extent of disease in patients before the after primary cytoreductive surgery. Magnetic resonance (MR) imaging is better reserved for problem solving when ultrasound findings are non-diagnostic or equivocal because, although it is more accurate for diagnosis, it is also more expensive.

The gold standard for diagnosis is cytohistopathological examination of specimen obtained from laparotomy or fine needle aspiration cytology of ovarian mass or cytology of ascetic fluid.

\section{Material \& Methods}

This is a prosective study conducted in the Department of Radiodiagnosis, G.S.V.M. Medical college \& L.L.R. Hospital, Kanpur, from December 2018 to November 2019. The study was conducted in collaboration with department of Obstetrics \& Gynecology.

The patients who fulfill the inclusion criteria from the outpatient department and from admissions in wards were enrolled in the study after taking informed consent.

\section{Sampling}

The study was conducted on 50 patients. Patients were selected from the outpatient department and from admissions in ward.

\section{Inclusion Criteria}

Patients with lower abdominal complaints will be screened with transabdominalsonography and in patients with suspected ovarian lesions detailed study by transvaginalsonography (TVS) was done after taking verbal and written consent, whenever possible in presence of patient's husband or attendant.

\section{Exclusion Criteria}

$>$ Unmarried female.

$>$ Patients not giving consent for TVS. 
Patients with narrow introitus or vagina who experience discomfort at attempted insertion of the transducer.

$>$ Extraovarian masses (Uterine or Broad ligament masses)

Ectopic Pregnancy.

\section{Method of study}

\section{Equipment}

All patients were examined on Samsung WS80A ultrasound color Doppler machine with 3.5 to $7 \mathrm{MHz}$ convex transducer and $6.5 \mathrm{MHz}$ tansvaginal transducer and with Gray scale, power and spectral Dopper. A 3.5 to $7 \mathrm{MHz}$ convex transducer is sometimes useful to assess large lesions as well as unmarried patients.

\section{Technique}

The patients were included from outpatient and inpatient section. A complete medical history and physical examination was done. Patient were examined with full bladder by transbdominal USG and in patient with adnexal masses detailed examination with transvaginal transducer was done.

The bladder is emptited before examination to bring the pelvic organs into the focal zone of the transvaginal transducer. The examination is explained to the patient \& verbal consent is obtained before beginning the examination. The transducer is prepared with ultrasound coupling gel \& then covered with a protective sheath condom. An external lubricant is then applied to the outside of the condom.

The transducer is inserted into the vagina with the patient supine, knees gently flexed \& hips elevated slightly on a pillow. The elevated hips allow free movement of transducer. With gentle rotation and angulation of the transducer both sagittal \& coronal images are obtained. Extreme angulation is applied to visualize the entire adnexae. Following the examination the transducer is removed from the vagina with the sheath intact. The sheath is then discarded $\&$ the transducer is cleaned to gel.
All adnexal masses were subjected to sonomorpholigical evaluation followed by blood flow analysis using color, spectral and power Doppler sonography. The examination was performed during the follicular proliferative phase of the menstrual cycle in permenopausal women.

Morphologic indexing of the adnexal masses was done using the Sassone score based on the visualization of inner wall structure and wall thickness, septae, solid parts and echogenicity. A mass with a score of 9 was classified as a high risk mass (suspicious for malignancy)

Subsequently, power and color Doppler flow imaging and spectral analysis were performed. Doppler paramenters were optimized for detection of flow and calculation of impedance indices. Flow results were reconded as being absent or present and further characterized as normal or increased, vessel location (peripheral, central, septal) and arrangement( regular/random).

On spectral Doppler, the lowest RI, PI and maximum PSV detected at any point in the mass was used for analysis. The Doppler findings were considered as being suggestive of malignancy when: $\mathrm{RI}<0.6$, PI $<1$ and $\mathrm{PSVmax}>15 \mathrm{~cm} / \mathrm{sec}$.

\section{Observations}

Present study was based on Folkman's theory of neovascularization, according to which malignant neoplasms elaborate a factor named Tumor Angiogenesis factor (TAF), which stimulates rapid formation of new capilaries. The study aimed at evaluating the efficacy of Color and Spectral Doppler in diagnosing the ovarian malignancy. It was carried out in the Department of Radiodiagnosis, G.S.V.M. Medical College. L.LR. Hospital, Kanpur from December 2018 to November 2019. The study was conducted in collaboration with department of Obstetrics \& Gynecology. The subjects for this study were 50 patients with 53 adnexal masses, including 3 cases of bilateral ovarian metastasis. Of these, 36 lesions were benign while 17 were malignant. 
Present study consisted of 50 patients, the youngest being of 18 years and oldest being, 70 years.

The peak age of incidence was in the 21-40 vears of age group with 34 subjects falling in this age group. The average age of the subjects being 36

A further classification of the age distrbution based on major pathologies was done. There was total of 36 benign lesions and malignant lesions (total 53 lesions including 3 bilateral) were noted.

Benign lesions of ovary were noted to be more common in the age group below 40 years making $31(86.11 \%)$ out of 36 cases. The cases i were clustered in 21-40 year with 29 out of the 36 making $(80.55 \%)$ of the cases.

Malignant lesions were noted in much older patients with 11 out of $17(64.7 \%)$ cases seen in patients with more than 40 vears of age. The cases were clustered in 51-70 year with 9 out of the 17 making $(52.94 \%)$ of the cases. The oldest patient was 70 years of age while the youngest was 18 years of age.

Abdominal pain was the chief complaint that the patient presented with in this series with 37 (74\%) out of 50 patients with this complaint. It was the commonest complain in both the benign \& malignant lesions.

Anorexia and weight loss is also a common complaint malignant lesions with 8 out of 14 patients $(57.1 \%)$ experencing it. Abdominal lump is also a common complaint in malignant lesions that 5 out of 14 (35.7\%) patients complained of it. In this study, out of 50 patients 37 patients were in Premenopausal state and 13 were in the postmenopausal state.

In the Premenopausal state benign lesions were more common, presenting 33 out of 37 (89.2\%) patients. In contrast to this, In the postmenopausal state malignant lesions were more common. 10 out of $13(77 \%)$ patients of postmenopausal age were having malignant lesions.

In this study, $36(67.92 \%)$ of the 53masses were benign and $17(32.07 \%)$ were malignant. Of these benign lesions, hemorrhagic cyst was the most common benign lesion presenting 12 (33.33\%) of cases. The second most common lesion is the mature teratoma making $22.22 \%$ of cases.

Other benign lesions include 4 Simple cysts. 3 endometriomas. 2 serous cystadenoma, 1 mucinous cystadenoma, 2 fibroma, 2 torsion, 1 ovarian hyperstimulation syndrome and 1 theca lutein cyst.

The most common malignant tumors in this study was serous stadenocarcinoma $(28.57 \%) \&$ second most common was ovarian metastasis $(21.42 \%)$. The primary malignancies in case of metastasis were breast carcinoma in 2 patients and gastric malignancy in 1 patient.

Other malignant lesions include 2 borderline serous tumors, 1 dyserminoma, 1 immature teratoma, 1 granulosa cell tumor, 1 endometroid carcinoma and 1 yolk sac tumor.

Morphologic indexing of the adnexal masses was done using the Sassone score based on the visualization of inner wall structure and wall thickness, septae, solid parts and echogenicity. A mass with a score of >_ 9 was classified as a high risk mass (suspicious for malignancy).

The sonomorphologic evaluation following Sassone scoring system had a sensitivity of $88.23 \%$, specificity $80.55 \%$, PPV $68.18 \%$ and NPV $93.54 \%$. 22 out of 53 masses were considered suspicious of malignancy (Sassone score >9) of which 15 were malignant (including bilateral ovarian metastasis as a separate lesion) and 7 were benign. High scoring (>_9) benign lesions were endometriomas, cystic teratomas, i mucinous cystadenomas, hemorrhagic cyst and fibromas. Non-suspicious sonomorphology (score<9) was noted in 31 mass lesions- 29 were benign and 2 were malignant ( 1 borderline serous tumor and 1 serous cystadenocarcinoma).

\section{Discussion}

In our study, the sonomorphologic evaluation following Sassone scoring system had a sensitivity of $88.23 \%$, specificity $80.55 \%$, PPV $68.18 \%$ and NPV 93.54. 22 out of 53 masses were considered suspiclous of malignancy (Sassone score >9) of which 15 were malignant and 7 were benign. Non- 
suspicious sonomorphology (score<9) was noted in 31 mass lesions- 29 were benign and 2 were malignant.

Guernero et al evaluated a total of 2148 pelvic masses in 1997 women in whom transvaginalsonography were performed with gray scale and color doppler, before surgical exploration and demonstrated that color doppler evaluation was more accurate in the diagnosis of adnexal malignancies in comparson with gray scale sonography because of a significantly higher specificity (94\% vs. 89\%o), with similar sensitivity ( $95 \%$ vs. $98 \%$ ).

Madan et al used the sassone scoring system for sonomorphologic evaluation of ovarian mass had a sensitivity of $92.3 \%$, specificity55.3\%. PPV $54.3 \%$.NPV 92.8. Forty six out of 74 masses were considered suspicious of malignancy (Sassone score> 9) of which 25 were malignant and 21 were benign. High scoring (>9) benign lesions were inflammatory adnexal masses, endometriomas, cystic teratomas, mucinous cystadenomas, and thecoma. Non-suspicious sonomorphology (score $<9$ ) was noted in 28 mass lesions- 26 were benign and 2 were malignant (1 immature teratoma and 1 serous cystadenocarcinoma).

Thus, sonomorphology has higher specificity in differentiating malignant from benign ovarian neoplasms if dermoids, inflammatory masses and endometriomas are excluded by recognizing their specific sonographic characteristics. Morphologic assessment by transvaginalsonography yielded additional information, especially small papillary projections $(<3 \mathrm{~mm})$, thick septae, and the relationship of the mass to surrounding structures. Papillary protrusions and abnormalities of the Inner wall structure were the most reliable findings associated with ovarian carcinoma.

The tumors evaluated with Color Doppler which showed presence of tumor neovasularisation in $94.11 \%$ of malignant tumors in contrast to $22.22 \%$ benign tumors.

The site of tumor vascularity did not affect the diagnosis in cystic neoplasm, as the tumor vascularity was encountered almost equally in the wall and septae. But, in solid malignant neoplasms; the central vascularity was encountered in $88.23 \%$ cases while peripheral vascularity was present in $17.64 \%$ cases.

In our study there was presence of tumor neovascularization in $94.11 \%$ of malignant tumors in contrast to $22.22 \%$ benign tumors.

Vascularisation in benign lesions tended to be peripheral and in malignant Lesionsit was central. In solid malignant neoplasms the central vascularity was encountered in $83.33 \%$ cases while peripheral vascularity was present in $16.66 \% \%$ cases.

KB Taoriet all evaluated the study group comprised of 60 patients with neoplastic ovarian masses. Color Doppler showed presence of tumor neovasularisation in $92.59 \%$ of malignant tumors in contrast to $42.24 \%$ benign tumors, Vascularisation in benign lesions tended to be peripheral and in malignant lesions it was central. In solid malignant neoplasms the central vascularity was encountered in $81.25 \%$ cases while peripheral vascularity was present in $18.75 \%$ cases. Absent blood flow in a solid tumor almost always ruled out the possibility of malignancy.

In the study by Madan et al, $92.5 \%$ (25/27 masses) malignant masses were vascularised as compared to $68.08 \%$ (32/47 masses) benign adnexal masses. Vascularisation in benign lesions tended to be peripheral and in malignant lesions it was central.

Thus, by comparison it can be stated that neovascularization is more common in malignant tumors as compared to benign tumors and it is central in malignant tumors and peripheral in benign tumors.

Malignant neoplasm offered lower resistance to blood flow due to presence of aberrant tumor vessels. Present study used a pre-established cutoff criterion of PI $<0.8$ and RI $<0.6$. In the present study, $82.35 \%$ of malignant tumors had PI $<0.8$ in contrast to only $8.33 \%$ of benign tumors. Similarly, $94.11 \%$ of malignant turnors showed RI $<0.6$ in contrast to only $11.11 \%$ of benign tumor. 
PSV was more than $15 \mathrm{~cm} / \mathrm{sec}$ in $15(88.23 \%)$ malignant tumors as compare $d$ to only $5.55 \%$ of benign tumors. A statistically significant difference was seen between PSV of benign and malignant lesions in close agreement to that observed by Valentin et al. Intratumoral PSV was hign in malignant lesions \& showed lesser degree of overlap.

When the data of present study was extrapolated using the criterion PI $<1.0 \&<$ RI 0.4 proposed by Kurjak et al \& Jean Noel Buy et al : 100\% of malignant tumors and $11.11 \%$ of benign tumors and $11.11 \%$ of benign tumors showed $\mathrm{PI}<1.0$ while only $23.52 \%$ malignant tumors \& none of the benign tumors showed RI $<0.4$.

The present study data was rendered slightly more specific (100\%) and less sensitive (23.52\%) with RI $<0.4$ and slightly more sensitive $(100 \%)$ and less specific $(88.88 \%)$ with $\mathrm{PI}<1$. Thus establishing the PI $<0.8 \&$ RI $<0.6$ as the better choice for the threshold values, in terms of optimizing the sensitivity and specificity. With using cut off criteria for PI 0.8 we got sensitivity of $82.35 \%$, specificity of $91.66 \%$, PPV of $82.35 \%$ and NPV of $91.66 \%$. While using $<0.6$ as a cut off for RI we got $94.11 \%$ sensitivity, $88.88 \%$ specificity, $80 \%$ PPV and $96.96 \%$ NPV. When $15 \mathrm{~cm} / \mathrm{sec}$ was considered as the cut-off value of peak systolic velocity, the sensitivity and specificity of peak systolic velocity in detecting malignant adnexal tumors were $88.23 \%$ and 94.44 $\%$, respectively. The specificity of the peak systolic velocity was significantly higher than that of the resistance index.

Our study also illustrated the special role of Color Doppler in labelling the solid tumor of ovary as benign; if it did not showed any significant vascularity. The definite diagnosis of malignancy in solid tumors was made only after the Color Doppler showed intratumoral vascularity (mainly central) and Spectral Doppler showed low resistance velocity waveforms in the intratumoral vessels.

In the present study, $82.35 \%$ of malignant tumors had PI $<0.8$ in contrast to only $8.33 \%$ of benign tumors. Similarly, $94.11 \%$ of malignant tumors showed RI $<0.6$ in contrast to only $11.11 \%$ of benign tumor. PSV was more than $15 \mathrm{~cm} / \mathrm{sec}$ in 15 $(88.23 \%)$ malignant tumors as compared to only $5.55 \%$ of benign tumors.

Our findings confirm those of most previous reports: malignant ovarian tumors tend to have lower PI and RI than do benign tumors.

Relatively high values of PI and RI usually indicate benignity. The PI value above which a mass will always be benign has been variably reported but, as in our study, usually has been found to be 0.8 or more.

The RI value above which a mass will always be benign has been reported as ranging from 0.4 to 0.6. The peak systolic velocity obtainedfrom intratumoral abnormal morphologic features provides a superior means for differentiating malignant from benign adnexal tumors.

With using cut off criteria for PI $<0.8$ we got sensitivity of $82.35 \%$, specificity of $91.66 \%$, PPV of $82.35 \%$ and NPV of $91.66 \%$.

While using $<0.6$ as a cut off for RI we got $94.11 \%$ sensitivity, $88.88 \%$ specificity, $80 \%$ PPV and $96.96 \%$ NPV. The present study yielded fairly good specificity and sensitivity with PI \& RI values of $<0.8 \&<0.6$ respectively, despite some overlap in the values between benign and malignant lesions.

Out of a total of 36 patients with benign tumors, $26(72.22 \%)$ patients were correctly diagnosed on gray scale USG alone, while 33(91.66\%) patients were correctly diagnosed when color and spectral Doppler were used along with the gray scale USG. Out of a total of 17 patients with malignant tumors, $12(70.58 \%)$ patients were correctly diagnosed on gray scale USG alone, while 15 (89\%o) patients were correctly diagnosed when color and spectral Doppler were used along with the B-mode USG.

In the present study, the prediction of benignity of tumor was more reliable with gray scale USG combined with Color Doppler and spectral Doppler as only $8.34 \%$ benign tumors were 
incorrectly diagnosed in contrast to $11 \%$ of malignant tumors.

The present study established the multifold increase in the Sensitivity, Specificity, Positive Predictive Value and Negative Predictive Value in establishing the pre-operative diagnosis of adnexal masses (especially in terms of benign and malignant); when using the gray scale USG in combination with color and spectral Doppler as compared to the gray scale USG alone.

\section{Conclusions}

In conclusion to our study, there is considerable overlap in the morphologic patterns of various adnexal masses and hence a multi-parameter analysis incorporating morphologic scoring, vessel location, vessel arrangement and spectral waveform analysis is helpful in determining those patients in whom early intervention is necessary.

Adding color Doppler to conventional sonography produced a specificity and positive predictive value higher than those of conventional sonography alone.

Ethical Approval: This research was cleared by ethical committee of GSVM Medical College, Kanpur

\section{Bibliography}

1. Block E. Quantitative morphological investigations of the follicular system in women. Jlc[u Eizdoc Ti/10/(Cope / m) $1951 ; 8: 33-54$.

2. Simkins CS. Development of the human ovary from birth to sexual maturity. /lm J llitat $1952 ; 51: 465-493$.

3. Gardner E, Gray D. I, O'Rahilly R. Female genital organs. Anatomy a regional study of human structure. 3r0 ed. Philadelphia, Pa Saunders. 1969 ; 490-502.

4. Bannister LH, Dyson M, Gray's anatomy : The anatomical basis of medicine and surgery. 38th ed Edinburgh, Scotland Cltm-c/Ti// Lir'ingsfol?e. 1995 ; 1 8611880 .
5. Hall DA. Sonographic appearance of the nomialovar $y$. of polycystic ovary disease and of functional ovarian cysts. Semin Ultrasound CT MR. 19834. 149-\ 65.

6. McLean JM. Embryology and anatomy of the ovaries. In : Fox H, Wells M, eds. Haines and Taylor obstetrical and gynaecological pathology. 4th ed. New York, NY :ClnwclzillLiviltg. \[olle. 1995 ; 673-698.

7. Gougeon A. Dynamics of follicular growth in the human: a model from preliminary results. HmnRejTrod 1986, 1 : 81-87.

8. Muradali D, Colgin T, Hayeems E, et al. Echogenic oval-ian foci without shadowing. Radio/ogy2002 ; 224 : 429435.

9. Leibman AJ, Kruse B, Mcsweeney MB. Transvaginalsonography comparison with transabdominal sonol\#aphy in the diagnosis of pelvic masses. Am J Roentgenot 1988, $1:$; $1: 89-92$.

10. Hall U, Mccarthy KA, Kopans IM. Sonographic Visualization of the nonnalpost menopausalovary.,/U///-(I. vomzd, l'fed 198h : 5 : 9-11.

11. Drake J. Diagnosis and management of the adnexal mass. /lm lftu71 P/n · sicitm 1998 ; 57( 10) : 2471-6, 2479-2480.

12. Cohen HL, Tice HM, Mandel FS. Ovarian v olume measured b!' US : bigger than we think. Radiolog $\backslash 1990 ; 177: 189-192$.

13. Kuliak A, Predanic M, Kupesic-U1-ek S, Jukic S. Transvaginal color and pulsed Doppler assessment ol'adnexal tumor $\mathrm{v}$ ascularity. G, vllecolOllcol 1993;503-9.

14. Lemer. JP, Timor-Tritsch IE, Feder man A, Abramov I ch G. Transvaginal ultrasonographic characterization of ovarianniasses with an improved, weighted scoring sytem. Allt J Qbs[e[ G\ · 11ecol $1994 ; 170: 81-85$.

15. Reles A, Wein U, Lichtenegger W. 7'ransvaginal color Dopplei- sonography and conventional sonography in the 
preoperative assessment of adnexal masses. J Clin Ultrasound 1997; 25: 21725.

16. Brown DL, Doubilet PM, Miller FH. Benign and malignant ovarian masses: selection of the most discriminating grayscale and Doppler sonographic features. Radiolo 1 998;208: 1 03-1 10.

17. Dock W, Grabenwoger F, Metz V, Eibenberger K, FaiTes MT. Tumor vascularization: assessment with duplex sonograph Radiology 1991 ; 181 : 241244.

18. Folkman J, Watson K, Ingber D, Hanahan D. Induction ol- angiogenesis during the transition from hyperplasia to neoplasiit. Nature 1 989, 339 : 58-61

19. Sassone AM, Timor-Tritsch IE, Artner A, Westhoff C, W'alTen WB. Transvaginal sonographic characterization of ovarian disease evaluation of a new scoring system to predict ovarian malignanc". Obstet Gynecol $199178 \cdot$. 0-76.

20. Hamper UM, Sheth S, Abhas FM, Rosenshei n NB. Aronson D. Kurman RJ. Transvaginal color Doppler sonograph o6 adnelal masses: differences in blood now impedance in benign and malignant lesions. Am J Roenlgenol 1993; 160 (6) : 1115-8. 